\title{
Serum cytokine profiles in patients with prostate carcinoma
}

\author{
ERI TAZAKI $^{1}$, NOBUAKI SHIMIZU ${ }^{2}$, RYOTA TANAKA $^{1}$, MASAKAZU YOSHIZUMI $^{3}$, \\ HIROSHI KAMMA ${ }^{4}$, SHIGERU IMOTO ${ }^{1}$, TOMOYUKI GOYA ${ }^{1}$, KUNIHISA KOZAWA ${ }^{3}$, \\ ATSUYOSHI NISHINA ${ }^{5}$ and HIROKAZU KIMURA ${ }^{6}$ \\ ${ }^{1}$ Department of Surgery, Kyorin University, School of Medicine, Mitaka-shi, Tokyo 181-8611; \\ ${ }^{2}$ Department of Urology, Gunma Prefectural Cancer Center, Ota-shi, Gunma 373-8550; \\ ${ }^{3}$ Gunma Prefectural Institute of Public Health and Environmental Sciences, Maebashi-shi, Gunma 371-0052; \\ ${ }^{4}$ Department of Pathology, Kyorin University, School of Medicine, Mitaka-shi, Tokyo 181-8611; \\ ${ }^{5}$ Yamagata Prefectural Yonezawa Women's Junior College, Yonezawa-shi, Yamagata 992-0025; \\ ${ }^{6}$ Infectious Disease Surveillance Center, National Institute of Infectious Diseases, \\ Musashimurayama-shi, Tokyo 208-0011, Japan
}

Received April 13, 2011; Accepted June 9, 2011

DOI: 10.3892/etm.2011.286

\begin{abstract}
It has been suggested that various cytokines are associated with the pathophysiology of prostate carcinoma (Pca). We profiled ten cytokines (IL-1 $\beta$, IL-2, IL-4, IL-5, IL-6, IL-8, IL-10, IL-12, IFN- $\gamma$ and TNF- $\alpha$ ) in the serum levels of 11 patients with organ-confined Pca, 15 with advanced Pca without cachexia, 8 with advanced Pca with cachexia (cachexia group) and 5 healthy males as controls. Cytokines were measured using a highly sensitive fluorescence microsphere system. Compared to the control group, serum levels of all cytokines were significantly higher in the cachexia group, and six cytokines (IL-1 $\beta$, IL-2, IL-8, IL-12, TNF- $\alpha$ and IFN- $\gamma$ ) were significantly higher in the group with advanced Pca without cachexia. In the group with organ-confined Pca, only IL-1 $\beta$ and IL-12 levels were significantly higher compared to the control group. In the cachexia group, levels of all cytokines apart from TNF- $\alpha$ were significantly higher compared to the group with organ-confined Pca, and levels of four cytokines (IL-2, IL-4, IL-8 and IL-10) were significantly higher compared to the group with advanced Pca without cachexia. These results indicate that i) an aberrance imbalance of cytokine production was associated with the pathophysiology of Pca and cachexia, ii) cytokine profiles in Pca patients were distinct by disease stage, and iii) IL-1 $\beta$ and IL-12 may be applicable as early diagnostic indicators.
\end{abstract}

\section{Introduction}

Prostate cancer (Pca) is a common cause of morbidity and mortality in the developed world, and is one of the major

Correspondence to: Dr Hirokazu Kimura, Infectious Disease Surveillance Center, National Institute of Infectious Diseases, 4-7-1 Gakuen, Musashimurayama-shi, Tokyo 208-0011, Japan

E-mail: kimhiro@nih.go.jp

Key words: cytokine, cachexia, prostate carcinoma, cytokine profiles malignant neoplasms in males (1-3). Sixty to seventy percent of patients with advanced Pca suffer from cachexia (4). Cancer cachexia is one of the most devastating conditions of advanced malignant disease. It is characterized by weight loss and anorexia that involves the depletion of host adipose tissue and skeletal muscle (5). Cachexia brings a lower quality of life, a poorer response to chemotherapy and reduced survival (6). However, as in patients with other types of malignant diseases, the pathophysiology and etiology of cachexia in patients with Pca is not well understood, and intervention strategies to alleviate the condition are not yet available.

The pathophysiology of cachexia associated with the progression of malignant disease in patients is multifactorial. Several cytokines, such as tumor necrosis factor $\alpha$ (TNF- $\alpha$ ), interleukin 1 (IL-1), IL-6 and IL-8, are known as agents that contribute to the condition (5). Cytokines are major homeostatic modulators in vivo and may be associated with the pathophysiology of various types of cancers (7-11). These cytokines are also reported to be associated with cachectic Pca $(5,6,12,13)$. For example, the levels of pro-inflammatory cytokine, TNF- $\alpha$, in cachectic Pca patients as well as organ-confined Pca patients were higher compared to the control (14). Another cytokine, IL-6, is also associated with the pathophysiology of cachexia in Pca (15). However, research into the relationships between other cytokines and the pathophysiology of cachexia is ongoing.

Previous studies have shown that more than thirty types of cytokines have been discovered and their biological functions studied (14-18). Each cytokine forms a cytokine network, and these networks may synchronize various biological events, including immunity, cell differentiation, proliferation and cancer growth in humans. Thus, the evaluation of various cytokines could be critical to gaining a better understanding of the pathophysiology of Pca, including the complication of cachexia. In addition, it has been reported that ceratin cytokines in the serum may be distinct in the disease stages of Pca $(11,13,14)$, although these reports involve less than five types of cytokines. Based on this background, we profiled ten types of cytokines in the sera levels of patients at various stages of Pca. 
Table I. Clinical data of the subjects.

\begin{tabular}{|c|c|c|c|c|}
\hline & $\begin{array}{l}\text { Control } \\
(\mathrm{n}=5)\end{array}$ & $\begin{array}{l}\text { Organ-confined Pca } \\
\qquad(\mathrm{n}=11)\end{array}$ & $\begin{array}{l}\text { Advanced Pca without } \\
\text { cachexia }(n=15)\end{array}$ & $\begin{array}{l}\text { Advanced Pca with } \\
\text { cachexia }(\mathrm{n}=8)\end{array}$ \\
\hline Age (years) & $49.4 \pm 3.3$ & $64.2 \pm 2.1$ & $70.7 \pm 2.0$ & $71.4 \pm 2.9$ \\
\hline Radical prostectomy & & 10 & & \\
\hline Hormone therapy & & 1 & 15 & 8 \\
\hline Stage I & & 11 & & \\
\hline Stage IV & & & 15 & 8 \\
\hline Total serum protein $(\mathrm{g} / \mathrm{dl})$ & $7.4 \pm 0.2$ & $7.2 \pm 0.1$ & $6.3 \pm 0.2$ & $5.6 \pm 0.3^{\mathrm{a}}$ \\
\hline Serum albumin $(\mathrm{g} / \mathrm{dl})$ & $4.1 \pm 0.1$ & $4.3 \pm 0.1$ & $3.5 \pm 0.1$ & $2.7 \pm 0.2^{\mathrm{a}}$ \\
\hline $\mathrm{Hb}\left(10^{4} / \mu \mathrm{l}\right)$ & $14.2 \pm 0.5$ & $14.3 \pm 0.5$ & $10.4 \pm 0.6^{\mathrm{a}}$ & $8.8 \pm 1.0^{\mathrm{a}}$ \\
\hline PSA (ng/ml) & $1.7 \pm 0.5$ & $11.6 \pm 3.0$ & $1,206.2 \pm 374.0^{\mathrm{a}}$ & $1,390.5 \pm 705.6^{\mathrm{a}}$ \\
\hline Life expectancy & All alive & All alive & 8.4 months & 1.2 months \\
\hline
\end{tabular}

Pca, prostate carcinoma. ${ }^{\mathrm{a}} \mathrm{p}<0.05$ vs. control or organ-confined Pca. Data are expressed as the means \pm SE. Details of the statistical method are described in the text.

\section{Materials and methods}

Subjects. Thirty-nine subjects were categorized into four groups as previously described (14): patients with organconfined Pca $(n=11)$, patients of advanced Pca without cachexia $(n=15)$, patients of advanced Pca with cachexia $(n=8)$ and healthy male adults as controls $(n=5)$ (Table I). Written informed consent was obtained from all patients. The study protocol was approved by the Ethics Committee on Human Research of Gunma Prefectural Cancer Center. The four groups were defined as follows.

Organ-confined Pca group. This group consisted of 11 patients with newly diagnosed, organ-confined Pca, at stage 1 . Radical prostectomy was performed on 10 of the 11 patients. The remaining patient underwent hormone therapy. Serum samples were obtained prior to primary therapy. The median age of this group was 64.2 years.

Advanced Pca without cachexia group. This group consisted of 15 advanced Pca patients without cachexia. They were all diagnosed with bone metastasis, but none of them suffered from peripheral edema, weight or appetite loss. The median age of this group was 70.7 years.

Advanced Pca with cachexia group (cachexia group). This group consisted of 8 advanced Pca patients with cachexia. They were all diagnosed with metastasis to other organs and suffered from appetite and weight loss. All patients died within 4 months (mean 1.2) after cachexia was diagnosed. The median age of this group was 71.4 years.

Control group. This group comprised 5 volunteers (healthy male adults) with no history of benign or malignant prostatic disease. The median age of this group was 49.4 years (Table I). Since high levels of prostate-specific antigen (PSA) in the serum due to benign prostatic hyperplasia (BPH) were found in individuals over the age of 51 years, we chose male subjects under 50 years of age as the control group $(19,20)$.

Measurement of serum cytokines. Serum specimens were obtained from the patients or the normal controls at the Gunma Prefectural Cancer Center. We evaluated the levels of ten cytokines in a small volume (50 $\mu \mathrm{l})$ of serum using a highly sensitive fluorescence microsphere system (Bio-Plex suspension array system, Hercules, CA, USA). Serum levels of the following cytokines were determined: IL-1 $\beta$, IL-2, IL-4, IL-5, IL-6, IL-8, IL-10, IL-12, IFN- $\gamma$ and TNF- $\alpha$. Samples were processed following the Bio-plex cytokine assay instruction manual. Total protein, albumin and hemoglobin were measured in each blood sample.

Categorization of cytokines. The ten cytokines were categorized into four groups as previously described (21): proinflammatory cytokines (IL- $1 \beta$, IL- 6 and TNF- $\alpha$; Fig. 1A-C), neutrophil recruitment-inducing chemokine (IL-8; Fig. 1D), Th1 cytokines (IL-2, IL-12 and IFN- $\gamma$; Fig. 1E-G) and Th2 cytokines (IL-4, IL-5 and IL-10; Fig. 1H-J).

Statistical analysis. Data were analyzed using the SAS JMP 8 software (Cary, NC, USA). All data were expressed as the means \pm SE. Statistical analysis of the cytokine levels was performed by the Kruskal-Wallis method. Statistical significance was set at the level of $\mathrm{p}<0.05$.

\section{Results}

Various cytokines (IL-1 $\beta$, IL-2, IL-4, IL-5, IL-6, IL-8, IL-10, IL-12, IFN- $\gamma$ and TNF- $\alpha$ ) were profiled in the serum levels of patients with organ-confined Pca ( $\mathrm{n}=11)$, advanced Pca without cachexia ( $\mathrm{n}=15)$, advanced Pca with cachexia (cachexia group, $\mathrm{n}=8$ ) and healthy males (control group, $n=5$ ). Fig. 1 shows the serum levels of the various cytokines quantitated in this study. Detailed statistical data with regard to cytokine concentrations in the sera are shown in Table II. Levels of all cytokines in the sera of the cachexia group were significantly higher than those in the control group. In the group with advanced Pca without cachexia, the levels of six cytokines (IL-1 $\beta$, IL-2, IL-8, IL-12, TNF- $\alpha$ and IFN- $\gamma$ ) were significantly higher than those in the control group, and IL-1 $\beta$ and IL-12 were significantly higher in the sera of the group with organ-confined Pca. Levels of all cytokines except TNF- $\alpha$ were significantly higher in the 


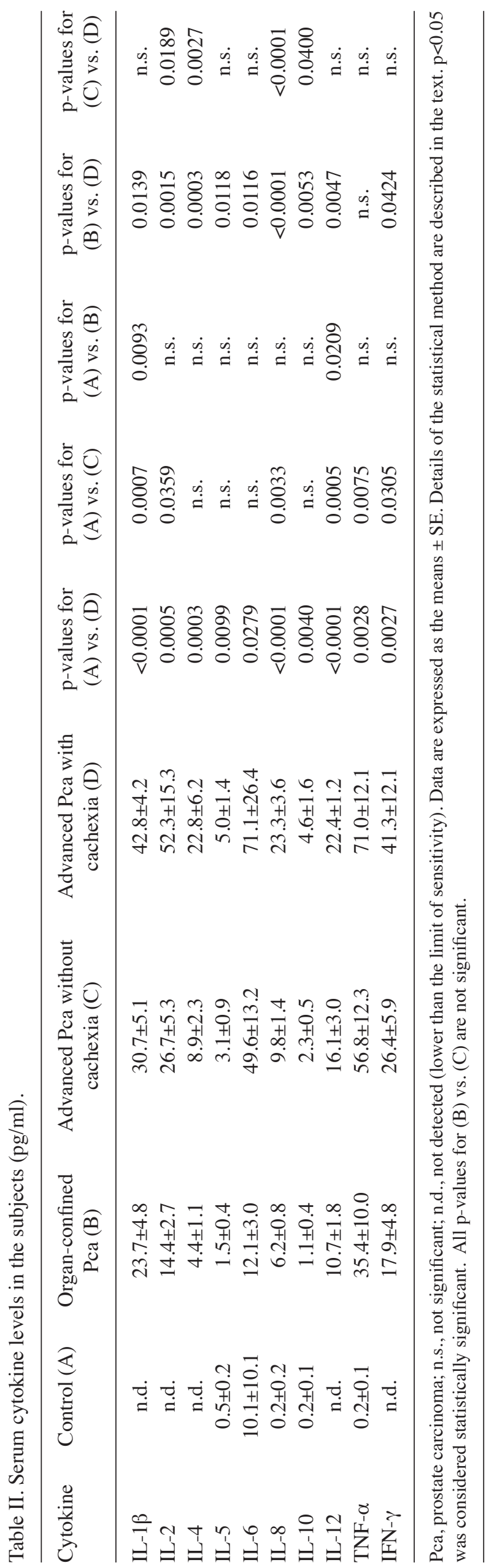

sera of the cachexia group than in the organ-confined Pca group. In addition, the levels of four cytokines (IL-2, IL-4, IL-8 and IL-10) in the cachexia group were significantly higher than those in the group with advanced Pca without cachexia.

Bood data were compared regarding serum total protein, serum albumin, serum PSA and hemoglobin concentrations in each group (Table I). The serum total protein and serum albumin in the cachexia group were lower than in the other groups. Hemoglobin concentrations of whole blood in the groups with advanced Pca without cachexia and cachexia were lower than those in both the organ-confined Pca and control groups. In addition, serum PSA levels in the group with advanced Pca without cachexia and cachexia group were higher than the level in the control group. However, no correlations between serum PSA and serum cytokine levels were found $(\mathrm{p}>0.05)$.

\section{Discussion}

We profiled serum cytokine levels (ten cytokines) in patients with organ-confined Pca, patients with advanced Pca without cachexia, patients with advanced Pca accompanied with cachexia (cachexia group) and healthy adults (control group). In the sera of the cachexia group, all cytokine levels were significantly higher than in the control group, nine cytokines (except TNF- $\alpha$ ) were significantly higher than in the organconfined Pca group and four cytokines (IL-2, IL-4, IL-8 and IL-10) were significantly higher than in the group with advanced Pca without cachexia. Compared to the controls, the levels of six cytokines (IL-1 $\beta$, IL-2, IL-8, IL-12, TNF- $\alpha$ and IFN- $\gamma$ ) were significantly higher in the group with advanced Pca without cachexia, but only two cytokines (IL-1 $\beta$ and IL-12) were significantly higher in the organ-confined Pca group. In addition, hemoglobin concentrations in blood, serum protein and serum albumin were lower in the cachexia group than these concentrations in the control group. These results suggest that aberrant and imbalanced production of various cytokines in Pca patients with or without cachexia may be associated with the pathophysiology of Pca, and these cytokine profiles may represent distinct disease stages in patients with Pca. Furthermore, the serum levels of IL-1 $\beta$ and IL-12 may be applicable biomarkers of early-stage Pca, although larger scale studies are required.

Previous reports suggest that various cytokine levels in Pca sera are higher than those in normal adults. For example, IL-1, IL-6, IL-8 and TNF- $\alpha$ levels were found to be higher than normal in patients with advanced Pca $(11,14)$. Indeed, our data are comparable to the data from these previous studies. Previous reports also discuss relationships between cytokinemia and the pathophysiology of Pca with or without cachexia. IL-6, IL- 8 and TNF- $\alpha$ have been particularly well studied $(5,14)$. The main symptoms of cachexia are weight loss and anorexia that involves the depletion of host adipose tissue and skeletal muscle. Both IL- 8 and TNF- $\alpha$ are involved in the depletion of adipose tissue. In addition, IL- 8 may be associated with tumor growth and the development of angiogenesis, tumorigenesis and metastasis $(22,23)$. Another report showed that IL-1 may play a similar role to TNF- $\alpha$ (24).

Anemia is a well-known complication in cachectic patients (11). A previous study suggested that TNF- $\alpha$ inhibits normal 

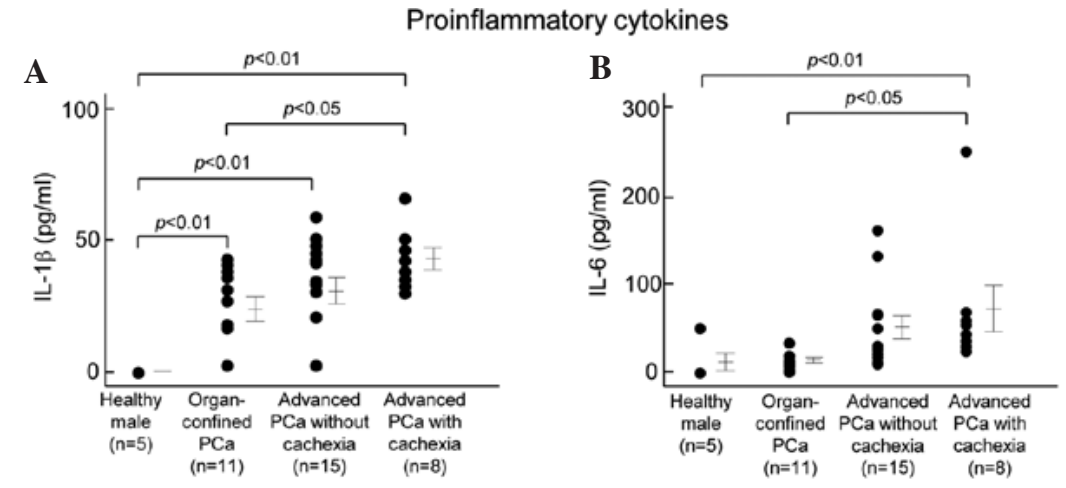

C Proinflammatory cytokines

D Neutrophil recruitment-inducing chemokine
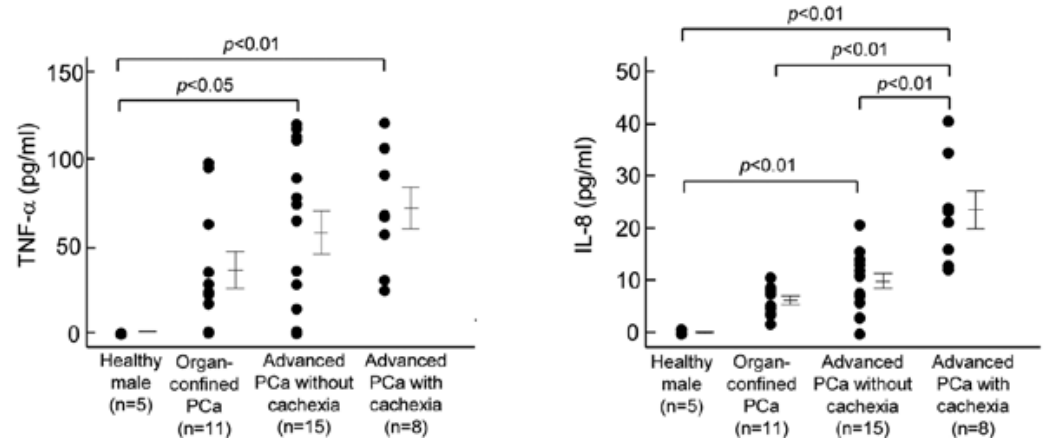

$\mathbf{E}$

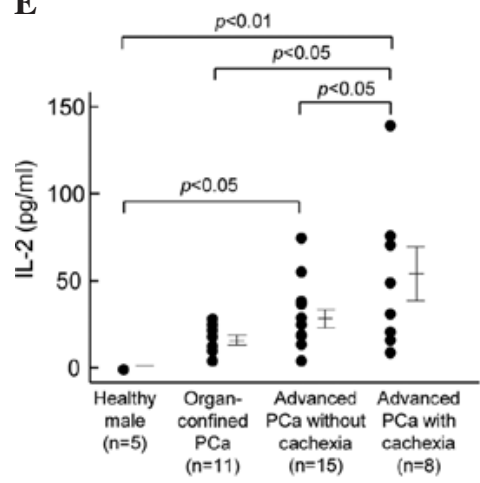

H

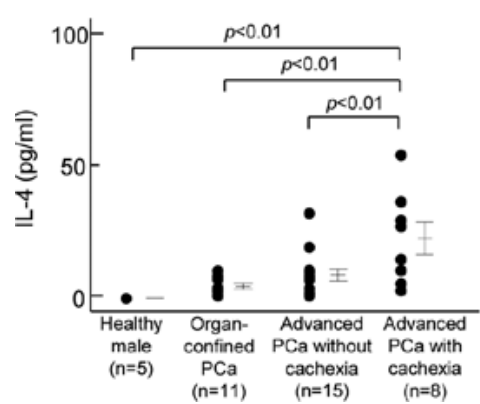

F

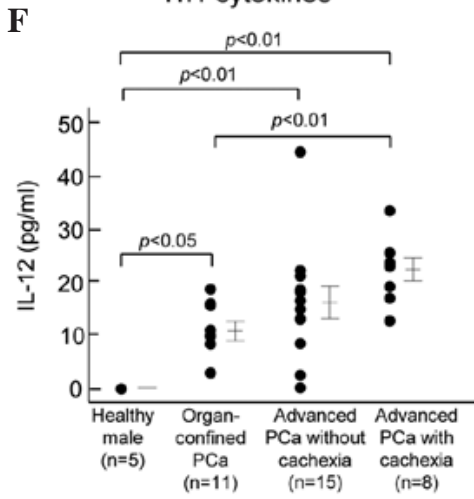

I

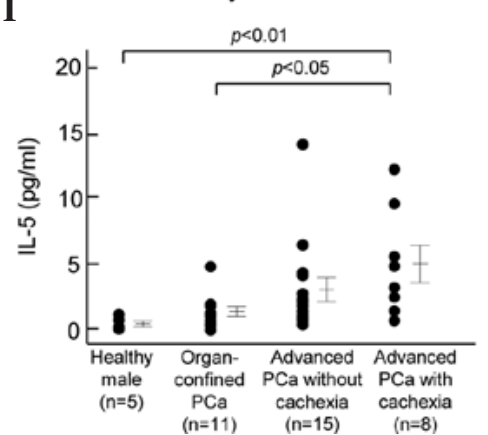

G

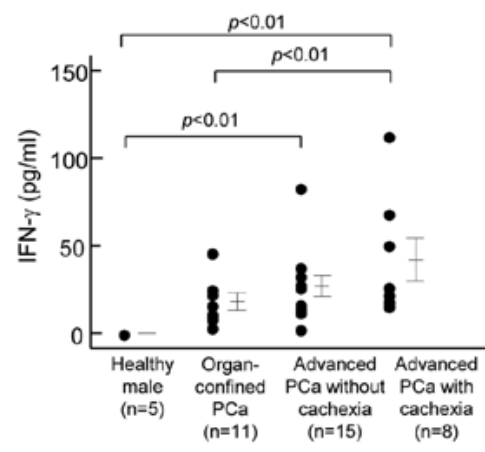

$\mathbf{J}$

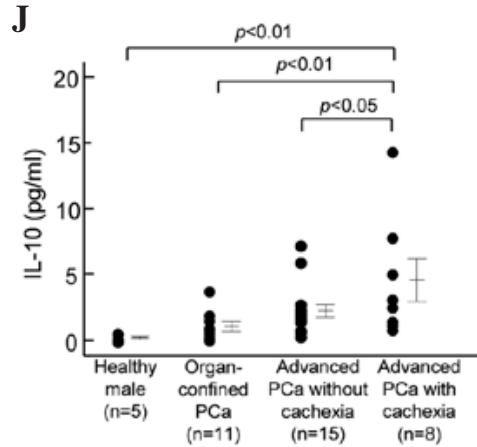

Figure 1. Concentrations of proinflammatory, neutrophil recruitment-inducing chemokine, Th1 and Th2 cytokines in the sera of healthy adults, and patients with organ-confined Pca, advanced Pca without cachexia and advanced Pca with cachexia. Detailed subject data and procedures for the determination of cytokine concentrations in the sera are given in Tables I and II. Vertical bars represent the means \pm SE.

hematopoietic progenitors, leading to both anemia and thrombocytopenia (25). Thus, in the present study, the high levels of these cytokines noted in cachectic patients may be associated with representative cachectic symptoms.
We also found that levels of other cytokines, such as IL-2, IL-4, IL-5, IL-10, IL-12 and IFN- $\gamma$, were higher than normal in cachexic patients. We explain the significances of these cytokines in Pca and cachexia as follows. First, IL-2, IL-12 
and IFN- $\gamma$ are Th1 cytokines and they may suppress cancer development and metastasis (26). These cytokines may be produced by phagocytic cells (monocytes, macrophages and neutrophils) and dendritic cells (DC), and activate natural killer (NK) cells (representative anticancer immune cells) leading to anticancer effects. IL-2 and IL-12 elicit IFN- $\gamma$ from NK cells, CD4-positive cells and CD8 cells, and accordingly play crucial anticancer effects in vivo (27). IL-12 and IFN- $\gamma$ may therefore be applicable to the development of therapies for various types cancers, including Pca (27). In the present study, the serum levels of IL-12 were significantly elevated in all groups with Pca, even in the organ-confined group (Table II). In addition, the serum levels of IFN- $\gamma$ were also elevated in the advanced Pca groups. Based on these results, it is suggested that elevated levels of Th1 cytokines, such as IL-2, IL-12 and IFN- $\gamma$, in Pca acted as anticancer agents, and cytokine profiles by disease stage of Pca were distinct.

IL-4, IL-5 and IL-10 are representative Th2 cytokines, and may be associated with cancer development. In particular, IL-4, IL-5 and IL-10 act as immunosuppressors of cancer immunity and promote cancer development $(28,29)$. In the present study, these immunosuppressive cytokines were significantly elevated in the cachexia group. Thus, these cytokines may have contributed to further cancer development.

More than 50 types of cytokines have been confirmed to date, and most of these are thought to be associated with cancer biology, such as cancer immunity and progression. Our preliminary results showed that many serum cytokine levels were elevated in patients with Pca, and cytokine profiles by disease stage of Pca were distinct. In addition, Th1 cytokines (IL-1 $\beta$ and IL-12) may be applicable diagnostic markers of early-stage Pca. Therefore, it is important to understand the diverse roles of various cytokines in order to further address the pathophysiology of various types of cancers, including Pca.

\section{Acknowledgements}

This study was supported in part by Research on Health and Welfare Programs from the Research Fund for Promoting Science and Technology of Gunma Prefecture, Japan.

\section{References}

1. Nelson WG: Prostate cancer prevention. Curr Opin Urol 17: 157-167, 2007

2. Eble JN, Sauter G, Epstein JI and Sesterhenn IA (eds): Pathology and Genetics of Tumours of the Urinary System and Male Genital Organs; World Health Organization Classification of Tumours. International Agency for Research on Cancer (IARC) Press, Lyon, 2004.

3. Parkin DM: Global cancer statistics in the year 2000. Lancet Oncol 2: 533-543, 2001

4. Argilés JM, Meijsing SH, Pallarés-Trujillo J, Guirao X and López-Soriano FJ: Cancer cachexia: a therapeutic approach. Med Res Rev 1: 83-101, 2001.

5. Tisdale MJ: Biology of cachexia. J Natl Cancer Inst 89: 1763-1773, 1997.

6. Davis MP and Dickerson D: Cachexia and anorexia: cancer's covert killer. Support Care Cancer 8: 180-187, 2000.

7. Smith KC, Bateman AC, Fussell HM and Howell WM: Cytokine gene polymorphisms and breast cancer susceptibility and prognosis. Eur J Immunogenet 31: 167-173, 2004.
8. Chavey C, Bibeau F, Gourgou-Bourgade S, Burlinchon S, Boissière F, Laune D, Roques S and Lazennec G: Oestrogen receptor-negative breast cancers exhibit high cytokine content. Breast Cancer Res 9: R15, 2007.

9. Suzuki S, Mita S, Kamohara H, Sakamoto K, Ishiko T and Ogawa M: IL-6 and IFN-gamma regulation of IL-10 production by human colon carcinoma cells. Int J Oncol 18: 581-586, 2001.

10. Szaflarska A, Szczepanik A, Siedlar M, Czupryna A, Sierzega M, Popiela $\mathrm{T}$ and Zembala M: Preoperative plasma level of IL-10 but not of proinflammatory cytokines is an independent prognostic factor in patients with gastric cancer. Anticancer Res 29: 5005-5012, 2009.

11. Kuroda K, Nakashima J, Kanao K, Kikuchi E, Miyajima A, Horiguchi Y, Nakagawa K, Oya M, Ohigashi T and Murai M: Interleukin 6 is associated with cachexia in patients with prostate cancer. Urology 69: 113-117, 2007.

12. Tisdale MJ: New cachexic factors. Curr Opin Clin Nutr Metab Care 3: 253-256, 1998.

13. Nakashima J, Tachibana M, Ueno M, Miyajima A, Baba S and Murai M: Association between tumor necrosis factor in serum and cachexia in patients with prostate cancer. Clin Cancer Res 7: 1743-1748, 1998.

14. Pfitzenmaier J, Vessella R, Higano CS, Noteboom JL, Wallace D Jr and Corey E: Elevation of cytokine levels in cachectic patients with prostate carcinoma. Cancer 97: 1211-1216, 2003.

15. Filella X, Alcover J, Zarco MA, Beardo P, Molina R and Ballesta AM: Analysis of type T1 and T2 cytokines in patients with prostate cancer. Prostate 44: 271-274, 2000.

16. Wang M, Hu Y and Stearns ME: A novel IL-10 signalling mechanism regulates TIMP-1 expression in human prostate tumour cells. Br J Cancer 88: 1605-1614, 2003.

17. Vindrieux D, Escobar P and Lazennec G: Emerging roles of chemokines in prostate cancer. Endocr Relat Cancer 3: 663-673, 2009.

18. Giri D, Ozen M and Ittmann M: Interleukin-6 is an autocrine growth factor in human prostate cancer. Am J Pathol 159: 2159-2165, 2001.

19. Bouraoui Y, Ricote M, García-Tuñón I, Rodriguez-Berriguete G, Touffehi M, Rais NB, Fraile B, Paniagua R, Oueslati R and Royuela M: Pro-inflammatory cytokines and prostate-specific antigen in hyperplasia and human prostate cancer. Cancer Detect Prev 32: 23-32, 2008.

20. Berry SJ, Coffey DS, Walsh PC and Ewing LL: The development of human benign prostatic hyperplasia with age. J Urol 132: 474-479, 1984.

21. Barnes PJ: The cytokine network in asthma and chronic obstructive pulmonary disease. J Clin Invest 118: 3546-3556, 2008.

22. Kim SJ, Uehara H, Karashima T, McCarty M, Shih N and Fidler IJ: Expression of interleukin-8 correlates with angiogenesis, tumorigenicity, and metastasis of human prostate cancer cells implanted orthotopically in nude mice. Neoplasia 3: 33-42, 2001.

23. Inoue K, Slaton JW, Eve BY, Kim SJ, Perrotte P, Balbay MD, Yano S, Bar-Eli M, Radinsky R, Pettaway CA and Dinney CP: Interleukin 8 expression regulates tumorigenicity and metastases in androgen-independent prostate cancer. Clin Cancer Res 6: 2104-2119, 2000.

24. Gelin J, Moldawer LL, Lönnroth C, Sherry B, Chizzonite R and Lundholm K: Role of endogenous tumor necrosis factor $\alpha$ and interleukin 1 for experimental tumor growth and the development of cancer cachexia. Cancer Res 51: 415-421, 1991.

25. Fandrey $J$ and Jelkmann WE: Interleukin- 1 and tumor necrosis factor $\alpha$ inhibit erythropoietin production in vitro. Ann NY Acad Sci 628: 250-255, 1991.

26. Del Vecchio M, Bajetta E, Canova S, Lotze MT, Wesa A, Parmiani $\mathrm{G}$ and Anichini A: Interleukin-12: Biological properties and clinical application. Clin Cancer Res 13: 4677-4685, 2007.

27. Sangro B, Melero I, Qian C and Prieto J: Gene therapy of cancer based on interleukin 12. Currt Gene Ther 5: 573-581, 2005.

28. Mocellin S, Marincola FM and Young HA: Interleukin-10 and the immune response against cancer: a counterpoint. J Leukoc Biol 78: 1043-1051, 2005 .

29. Quatan N, Meyer B, Bailey M and Pandha H: Persistently high levels of immunosuppressive cytokines in patients after radical prostatectomy. Prostate Cancer Prostatic Dis 9: 420-425, 2006. 